\title{
Dissolution of Silver Nanowires and Nanospheres Dictates Their Toxicity to Escherichia coli
}

\author{
Meeri Visnapuu, ${ }^{1}$ Urmas Joost, ${ }^{1,2}$ Katre Juganson, ${ }^{3,4}$ Kai Künnis-Beres, ${ }^{3}$ Anne Kahru, ${ }^{3}$ \\ Vambola Kisand, ${ }^{1,2}$ and Angela Ivask ${ }^{3}$ \\ ${ }^{1}$ Institute of Physics, University of Tartu, Riia 142, 51014 Tartu, Estonia \\ ${ }^{2}$ Estonian Nanotechnology Competence Center, Riia 142, 51014 Tartu, Estonia \\ ${ }^{3}$ Laboratory of Environmental Toxicology, National Institute of Chemical Physics and Biophysics, Akadeemia 23, 12618 Tallinn, Estonia \\ ${ }^{4}$ Department of Chemistry, Tallinn University of Technology, Akadeemia 15, 12618 Tallinn, Estonia
}

Correspondence should be addressed to Angela Ivask; angela.ivask@kbfi.ee

Received 30 April 2013; Revised 1 July 2013; Accepted 8 July 2013

Academic Editor: Muthuswamy Sathishkumar

Copyright (c) 2013 Meeri Visnapuu et al. This is an open access article distributed under the Creative Commons Attribution License, which permits unrestricted use, distribution, and reproduction in any medium, provided the original work is properly cited.

\begin{abstract}
Silver nanoparticles are extensively used in antibacterial applications. However, the mechanisms of their antibacterial action are not yet fully explored. We studied the solubility-driven toxicity of $100 \times 6100 \mathrm{~nm}$ (mean primary diameter $\times$ length) silver nanowires (NWs) to recombinant bioluminescent Escherichia coli as a target representative of enteric pathogens. The bacteria were exposed to silver nanostructures in water to exclude the speciation-driven alterations. Spherical silver nanoparticles $(83 \mathrm{~nm}$ mean primary size) were used as a control for the effect of NPs shape. Toxicity of both Ag NWs and spheres to E. coli was observed at similar nominal concentrations: the $4 \mathrm{~h}$ EC50 values, calculated on the basis of inhibition of bacterial bioluminescence, were $0.42 \pm 0.06$ and $0.68 \pm 0.01 \mathrm{mg} \mathrm{Ag} / \mathrm{L}$, respectively. Dissolution and bioavailability of $\mathrm{Ag}$ from NWs and nanospheres, analyzed with AAS or Ag-sensor bacteria, respectively, suggested that the toxic effects were caused by solubilized $\mathrm{Ag}^{+}$ions. Moreover, the antibacterial activities of Ag NWs suspension and its ultracentrifuged particle-free supernatant were equal. The latter indicated that the toxic effects of $\sim 80-100 \mathrm{~nm}$ Ag nanostructures to Escherichia coli were solely dependent on their dissolution and no shape-induced/related effects were observed. Yet, additional nanospecific effects could come into play in case of smaller nanosilver particles.
\end{abstract}

\section{Introduction}

Nanoparticles are included in various consumer goods. The most widely used are silver nanoparticles (Ag NPs) which, due to their antimicrobial effects $[1,2]$, are being incorporated into medical equipment coatings, cosmetic products, textiles, sprays, and other products (http://www.nanotechproject.org/). In addition to antimicrobial activity, Ag NPs possess characteristics that enable their use in electronics [3], solar energy conversion [4], or biosensing [5]. Current synthesis methods enable the production of differently shaped and sized Ag NPs. While mostly spherical Ag nanoparticles are produced, Ag nanowires have found increasing applications in different technologies, for example, in circuits to be used in nanoelectronic applications [6] or in solar cells [4].
The widespread use of Ag NPs has raised a concern about their environmental release and the subsequent effects. It has been clearly shown that studies on ecotoxicological effects of nanomaterials, including silver, drastically lag behind the design of new nanomaterials [7]. From the current studies it is clear that Ag NPs exhibit toxic effects to key organisms of the aquatic environment $[8,9]$. These effects have mostly been explained by a combination of dissolved $\mathrm{Ag}^{+}$ions and specific nanoeffects [10]. In case of bacteria, toxicity of $\mathrm{Ag}$ NPs has been shown to be driven by (i) attachment to the surface of the cell membrane and the following disturbance of membrane permeability and respiration [11], (ii) penetration of the cell membrane of the bacteria (1-10 $\mathrm{nm} \mathrm{Ag}$ particles) and induction of subsequent physiological effects [12], and (iii) release of silver ions [13-15]. Although the released silver 
ions have been considered the main drivers for Ag NPs' antibacterial effects, it has been suggested that the extent of dissolution depends on nanoparticles' size and surface area as well as on the shape. Despite a large number of toxicological and ecotoxicological studies available for $\mathrm{Ag}$ NPs, most of these have been conducted using spherical particles. Only a few studies have explored the toxic effects of distinct shapes of nanosized Ag. Pal et al. [16] showed that among spherical, truncated triangular (both $\sim 40 \mathrm{~nm}$ in diameter) and rod-shaped ( $\sim 100 \mathrm{~nm}$ in length and $10 \mathrm{~nm}$ in diameter) Ag NPs the triangular Ag nanoplates had the most pronounced antibacterial effects. They suggested that the truncated particles exhibited higher area of active facets with $\{111\}$ lattice plane which has high biological reactivity. In similar line, Sadeghi et al. [17] reported that among differentially shaped monodisperse Ag nanoparticles, nanoplates exhibited the highest toxicity to Escherichia coli and Streptococcus mutans. The same study also showed that $\mathrm{Ag}$ nanorods were more toxic to bacteria than $\mathrm{Ag}$ nanospheres. Yet, as particle sizes were not reported in this study, no conclusion on whether the differential toxicity was indeed caused by different shapes of Ag nanoparticles or their different sizes could be drawn. Another study by Ashkarran et al. [18] showed that spherical (17 $\mathrm{nm}$ hydrodynamic size) and rod-shaped ( $92 \mathrm{~nm}$ hydrodynamic size) Ag particles had similar toxicity to E. coli and Bacillus subtilis, while triangular Ag plates (50 nm hydrodynamic size) were less toxic. Shapedependent toxicity of nanoparticles to bacterial cells has also been reported for other NPs than silver. For example, Liu et al. [19] reported that rod-shaped carbon structures exhibited antibacterial activity as these particles punctured bacterial cells. Thus, in general, the currently available studies indicate that the toxic effects of Ag nanoparticles may be increased by changing the shape of the particles from spherical to wires or triangles. This suggestion is supported by the few existing shape-related toxicity studies on cell cultures. Changing the shape of Ag particles from spherical to triangular and rodshape was shown to increase the toxicity of Ag NPs to fish gill epithelial cells and zebrafish embryos [20] and to human alveolar epithelial cells [21]. Thus, it could be hypothesized that the rod/wire-shaped Ag nanoparticles could integrate the antimicrobial effects of silver with the inherent toxicity of nanosized wires. To test that hypothesis, we studied the antibacterial effects of silver nanowires $(100 \mathrm{~nm}$ in diameter and $6100 \mathrm{~nm}$ in length) to a bioluminescent Escherichia coli strain as a surrogate or representative of closely related enteric opportunistic pathogens in humans. As a control for the shape, silver nanospheres with similar particle diameter $(83 \mathrm{~nm})$ were tested in parallel. The contribution of $\mathrm{Ag}^{+}$ ions to overall toxicity was evaluated by determining the dissolution of the studied Ag NPs using atomic absorption spectroscopy (AAS). From the results it was evident that the toxicity and bioavailability of both wire-shaped and spherical nanoparticles to $E$. coli were dependent on nanoparticles dissolution. Although particle dissolution explained the toxic effects of the relatively large Ag nanowires used in this study, smaller nanowires may also exhibit shape-related cellular effects.

\section{Materials and Methods}

2.1. Materials. Water purified with MilliQ equipment (Millipore, USA) and reagent grade chemicals were used throughout the experiments. Components of bacterial growth media were purchased from LabM (UK). Ampicillin sodium salt and tetracycline hydrochloride were purchased from SigmaAldrich. $\mathrm{AgNO}_{3}$ (used as the positive control for $\mathrm{Ag}^{+}$ions due to its $100 \%$ dissolution in water) was purchased from J.T Baker (USA). $0.05 \mathrm{M}$ stock solution in water was used for toxicological experiments. Ag nanowires (NWs) were purchased from Seashell Technology (USA) as suspensions in isopropanol. Before the test, Ag NWs were dried and redispersed in water at $5000 \mathrm{mg} / \mathrm{L}$ using Digital Sonifier 450 (Branson Ultrashell, Germany) probe sonicator at $40 \mathrm{~W}$ for $1.5 \mathrm{~min}$. Ag nanospheres (83 $\mathrm{nm}$ average primary size; citrate stabilized) were purchased as aqueous suspensions (72 mg/L) from MKNano (Canada). Before the test, the stock suspension of Ag nanospheres was sonicated as described above but for $2.5 \mathrm{~min}$. Both $\mathrm{Ag} \mathrm{NWs}$ and $\mathrm{Ag}$ nanospheres were diluted in water to the desired concentration. Exact concentrations of Ag NWs and spheres in the tests were determined using GF-AAS in a certified laboratory.

2.2. Nanoparticle Characterization. Electron spectroscopic images from Ag particles were taken using SEM-FIB-EDX instrument (FEI Helios NanoLab 600, USA). SEM samples were prepared by depositing drops of aqueous suspensions of Ag nanoparticles on a silicon wafer. The samples were allowed to dry overnight at room temperature. The images were taken using an electron beam energy corresponding to $15 \mathrm{kV}$ high voltage. 20 particles were measured from images using image processing software ImageJ (http://rsbweb.nih.gov/ij/) to obtain the primary dimensions of the nanostructures. Elemental analysis of the particles was performed using energy-dispersive X-ray spectroscopy (EDX) function (Oxford Instruments, UK) of the FIB-SEMEDX setup. The EDX measurements were conducted by using primary electron beam with an acceleration voltage of $15 \mathrm{kV}$ to detect $\mathrm{Ag} \mathrm{L} \alpha$ X-ray fluorescence (2.98 keV) [22]. The hydrodynamic diameter and surface charge (zeta potential) of $\mathrm{Ag}$ nanospheres $(15 \mathrm{mg} / \mathrm{L})$ and $\mathrm{Ag}$ nanowires $(17 \mathrm{mg} / \mathrm{L})$ were measured using Zetasizer Nano ZS (Malvern Instruments, UK) using dynamic light scattering (DLS) and electrophoretic light scattering (ELS) functions, respectively. DLS results are applicable for spherical particles only (User manual from Malvern Instruments) and thus were not used for Ag NWs. ELS results were used for Ag nanospheres as well as for Ag NWs. UV-Vis absorption spectra of Ag NWs $(100 \mathrm{mg} / \mathrm{L})$ and $\mathrm{Ag}$ nanospheres $(10 \mathrm{mg} / \mathrm{L})$ aqueous suspensions were recorded using a dual-beam spectrophotometer Multiskan Spectrum (Thermo Scientific) in 300$600 \mathrm{~nm}$ wavelength range as suggested by Chen et al. [23], Evanoff and Chumanov [24], and Yang et al. [25]. pH of the Ag NWs and Ag spheres suspensions was measured with $\mathrm{pH}$ meter Orion 8220 BNWP (Thermo Scientific, USA).

Dissolution of $\mathrm{Ag}^{+}$ions from $\mathrm{Ag}$ nanostructures was analyzed in the suspensions equal to the highest concentration used in toxicological tests: $36 \mathrm{mg}$ of $\mathrm{Ag}$ spheres/L 
and $15 \mathrm{mg}$ Ag NWs/L. After preparation, the suspensions were incubated at room temperature for $4 \mathrm{~h}$ (the time duration also used for the bacterial toxicity assay) and then ultracentrifuged at $390000 \mathrm{~g}$ for 45 minutes as suggested by Bondarenko et al. [15] and $\mathrm{Ma}$ et al. [26], to separate the particulate and ionic fraction. The carefully removed supernatant was analyzed for Ag using GF-AAS in a certified laboratory. Part of the supernatant was also used for the toxicity assay as described below.

2.3. Toxicity and Bioavailability Tests Using Recombinant Escherichia coli. Two recombinant bioluminescent Escherichia coli strains were used (Table 1): toxicity was analyzed using constitutively bioluminescent bacterium $E$. coli MC1061(pSLlux) and bioavailability of silver ions $\left(\mathrm{Ag}^{+}\right)$ released from $\mathrm{Ag}$ nanostructures was measured using a $\mathrm{Ag}^{+}$induced bacterium E. coli MC1061(pSLcueR/pDNcopAlux).

Before the test, the bacteria were cultivated in LB medium (per litre: $10 \mathrm{~g}$ tryptone, $5 \mathrm{~g}$ of yeast extract and $\mathrm{NaCl}$ ) supplemented with $100 \mu \mathrm{g} / \mathrm{mL}$ of ampicillin (bioluminescent E. coli) or with $100 \mu \mathrm{g} / \mathrm{mL}$ of ampicillin and $10 \mu \mathrm{g} / \mathrm{mL}$ of tetracycline $\left(\mathrm{Ag}^{+}\right.$-induced E. coli) to OD600 of 0.6. Then, the cells were washed twice with water by repeating the following cycle: centrifugation of the cells at $5000 \mathrm{~g}$, removing the supernatant and adding similar volume of water. For toxicity and bioavailability assays, the OD600 of the bacterial culture was adjusted with water to 0.2 (bioluminescent $E$. coli) or $0.4\left(\mathrm{Ag}^{+}\right.$-induced E. coli). $100 \mu \mathrm{L}$ of serially diluted aqueous suspensions of $\mathrm{Ag}$ nanowires, $\mathrm{Ag}$ nanospheres, their ultracentrifuged supernatants, and $\mathrm{AgNO}_{3}$ (solubility control for both nanostructures) were transferred to white microplate (polystyrene, Greiner) wells, each concentration in two replicates. Water was used as the negative control. Then, $100 \mu \mathrm{L}$ of bacterial suspension was added to each well and the plate was incubated in the dark at room temperature (about $20^{\circ} \mathrm{C}$ ). Bioluminescence of the bacteria was registered at least every hour during 4 hours using Microplate Luminometer Orion II (Berthold Detection Systems). The 4-hour incubation time was chosen as a compromise: during that relatively long exposure period significant induction of the luminescence of the $\mathrm{Ag}^{+}$-induced $E$. coli was observed (Figure 1(b)) yet no effect on the sensitivity of the constitutively luminescent bioluminescent E. coli was recorded (Figure 1(a)). All the experiments were carried out over two or three separate days in order to take into account the inherent variability of the bioassays.

Inhibition of bioluminescence of bioluminescent $E$. coli was calculated by the following formula:

$$
\text { Inhibition }(\%)=100-\frac{\mathrm{RLU}_{S}}{\mathrm{RLU}_{B}} \times 100 \text {, }
$$

where $\mathrm{RLU}_{S}$ is bacterial bioluminescence in the sample and $\mathrm{RLU}_{B}$ is bacterial bioluminescence in water. EC50 was calculated by plotting the $\log _{10}$ values of Ag concentrations against bioluminescence inhibition using GraphPad program.
Induction of the $\mathrm{Ag}^{+}$-induced $E$. coli was calculated by the following formula:

$$
\text { Induction }(\text { fold })=\frac{\mathrm{RLU}_{S}}{\mathrm{RLU}_{B}} \text {. }
$$

Induction value of 2 was considered as induction threshold as suggested by Hakkila et al. [29]. Bioavailability of Ag to sensor bacteria was calculated from induction threshold. Both EC50 and bioavailability values were normalized to dissolved Ag, determined from the ultracentrifuged extracts of Ag nanostructures by GF-AAS.

In addition to bioluminescence inhibition, the impact of Ag nanostructures on bacterial viability was also evaluated. Once the bacteria had been exposed to Ag NWs for 4 hours for bioluminescence measurement, $2 \mu \mathrm{L}$ of the bacteriasample mixture was pipetted onto LB agar plates and the plates were incubated at $30^{\circ} \mathrm{C}$ for 24 hours. The potential of bacterial cells to form colonies on LB plates after being exposed to AgNWs for 4 hours was visually evaluated.

2.4. Statistical Analysis. 95\% confidence intervals of EC50 and induction values were calculated using the program GraphPad. MS Excel was used to calculate standard deviations and perform $t$-test.

\section{Results and Discussion}

3.1. Characterization of Ag Nanowires and Spheres. Electron micrographs of Ag nanowires (Ag NWs) and spherical particles that were used as control nanoparticles (NPs) are shown in Figure 2. EDX point analysis made to the regions indicated with the letter " $p$ " in Figure 2 confirmed that particles only consisted of Ag. Ag NWs were $70-150 \mathrm{~nm}$ in diameter and 3000-8000 $\mathrm{nm}$ in length (Table 2). The diameter of Ag spheres according to SEM measurements was $50-100 \mathrm{~nm}$. The average hydrodynamic diameter of Ag spheres in water, based on DLS analysis, was $98 \mathrm{~nm}$ (Table 2). EDX mapping images (Figure 2) indicate that the observed nanostructures only contained $\mathrm{Ag}$ in addition to $\mathrm{Si}$ and $\mathrm{C}$ signals that originated from the underlying sample holder. UV-vis spectra of Ag NWs and spheres are shown in Figure 2. As expected, the absorption peak of spherical particles was narrower than that of NWs. Similar result was shown earlier by Ashkarran et al. [18]. Absorption peak of Ag NWs at $380 \mathrm{~nm}$ corresponds to transverse plasmon mode of Ag NWs, and the shoulder peak at $\sim 355 \mathrm{~nm}$ can be attributed to long nanowires which exhibit similar optical properties to bulk silver [30].

The surface charge of both nanostructures was highly negative $(-36$ and $-46 \mathrm{mV}$ ) (Table 2$)$. Also, both nanostructures dissolved at similar rate: $2.4 \%$ of $\mathrm{Ag}$ NWs and $2.2 \%$ of $\mathrm{Ag}$ spheres were in the form of $\mathrm{Ag}^{+}$ions in the aqueous suspensions of these nanostructures (Table 2).

The relatively similar primary particle diameter and negative surface charge as well as similar dissolution rate (Table 2) supported the use of Ag nanospheres as controls for Ag NWs shape in further experiments. 


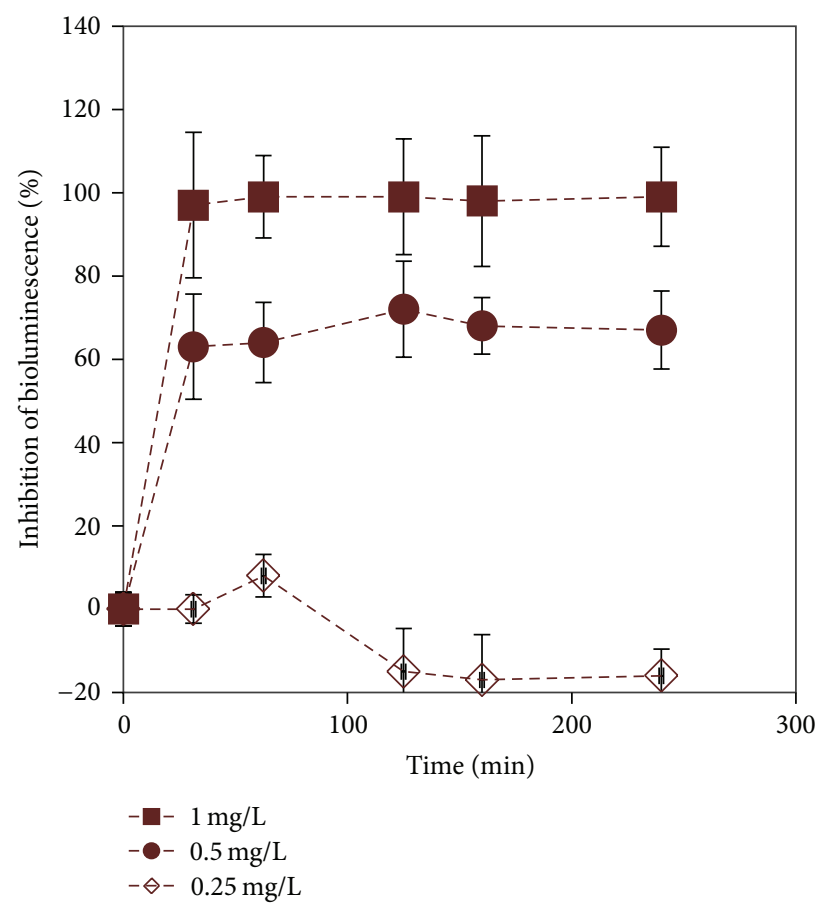

(a)

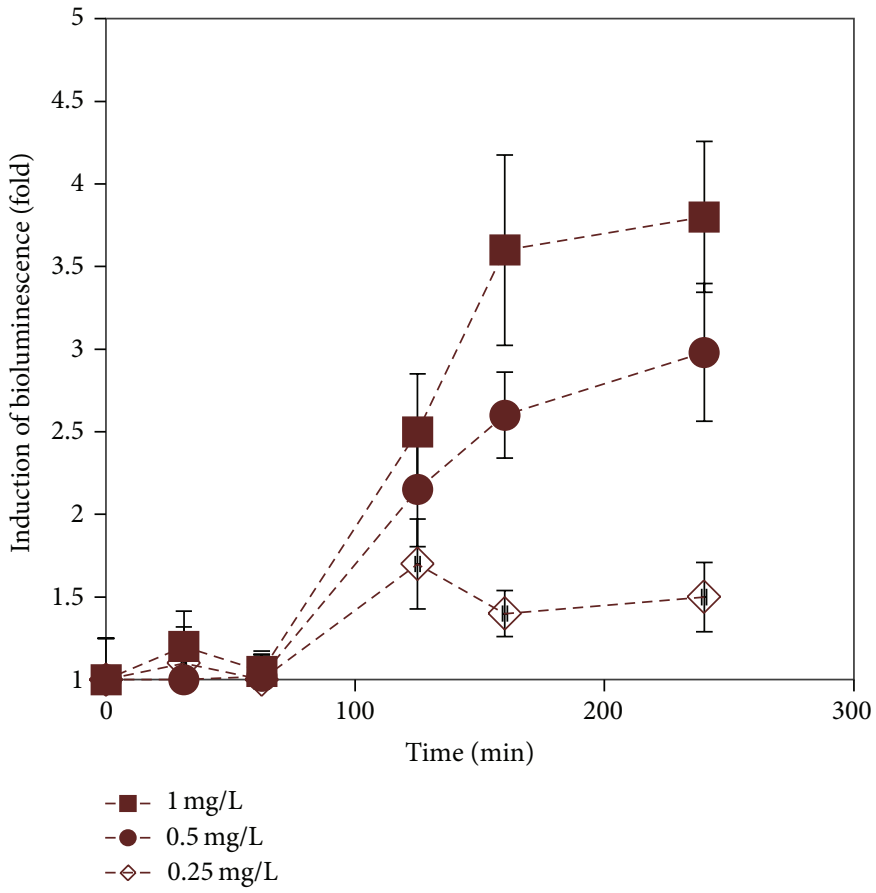

(b)

FIGURE 1: (a) Inhibition of bioluminescence (\% of the control) in bioluminescent Escherichia coli in time and (b) induction of bioluminescence in $\mathrm{Ag}^{+}$-induced Escherichia coli in time upon exposure to different nominal concentrations of $\mathrm{Ag}$ nanowires (concentrations shown on the graphs). Note that the significant induction of $\mathrm{Ag}^{+}$-induced $E$. coli was observed only since $120 \mathrm{~min}$ of exposure and the induction increased with time. $240 \mathrm{~min}$ incubation was chosen for further experiments as this time resulted in highly significant induction, and the signal was easily distinguishable from the background (not induced) bioluminescence. Data represent mean \pm standard deviation of three experiments. Negative inhibition values in (a) indicate small increase of bacterial bioluminescence at relatively low concentrations of Ag NWs, likely due to the hormesis effect [28].

TABLE 1: Characteristics of the applied Escherichia coli strains.

\begin{tabular}{|c|c|c|c|}
\hline & Characteristics & Designation in the current study & Reference \\
\hline E. coli MC1061 (pSLlux) & $\begin{array}{l}\text { Constitutively bioluminescent; } \\
\text { bioluminescence is decreased in response to } \\
\text { interference in cellular energy production, } \\
\text { effects on membranes, or decreased viability }\end{array}$ & Bioluminescent $E$. coli & {$[27]$} \\
\hline E. coli MC1061 (pSLcueR/pDNcopAlux) & $\begin{array}{l}\mathrm{Ag}^{+} \text {-induced strain; bioluminescence is } \\
\text { increased in response to intracellular } \mathrm{Ag}^{+} \text {ions }\end{array}$ & $\mathrm{Ag}^{+}$-induced E. coli & {$[27]$} \\
\hline
\end{tabular}

3.2. Toxicity and Bioavailability of Ag Nanowires to Escherichia coli. Inhibition of light output of bioluminescent $E$. coli in response to its exposure to Ag nanostructures and comparatively to $\mathrm{AgNO}_{3}$ as a solubility control is shown in Figure 3(a). The respective EC50 values are indicated in Figure 3(b). The EC50 values of Ag NWs and spherical Ag nanoparticles were $0.42 \pm 0.06$ and $0.68 \pm 0.01 \mathrm{mg} \mathrm{Ag} / \mathrm{L}$, respectively (Figure 3 ). In parallel experiments the EC50 value for $\mathrm{AgNO}_{3}$ (control for dissolved $\mathrm{Ag}^{+}$ions) was $0.0082 \mathrm{mg} / \mathrm{L}$. Taking into account the dissolution of Ag NWs and spheres (Table 2), the calculated amount of dissolved $\mathrm{Ag}^{+}$ions at EC50 concentrations of Ag NWs and spheres was 0.011 and $0.015 \mathrm{mg} / \mathrm{L}$, respectively (Figure 3(b), open columns). These values were not statistically significantly different from the EC50 value of $\mathrm{AgNO}_{3}$ and thus, suggest that the observed toxic effects of Ag NWs and spheres were driven by dissolved $\mathrm{Ag}^{+}$ions. Further evidence that Ag NWs and spheres exhibited their toxicity via dissolved metal ions was obtained using $\mathrm{Ag}^{+}$-induced bacterial cells. When exposed to Ag NWs and spheres, the $\mathrm{Ag}^{+}$-induced bacteria were induced by similar nominal concentrations, $0.46 \pm 0.2$ and $0.44 \pm 0.16 \mathrm{mg} \mathrm{Ag} / \mathrm{L}$, respectively (Figures 3(c) and 3(d)) indicating that similar amount of $\mathrm{Ag}^{+}$ ions was released from Ag nanoparticles and entered bacterial cells. Again, when the nominal inducing concentrations of Ag NWs and spheres were corrected for dissolution, the dissolved concentration of $\mathrm{Ag}$ in the induction threshold of the $\mathrm{Ag}^{+}$-induced bacteria was statistically similar in case of all Ag formulations (Figure 3(d)).

Additional evidence that dissolved $\mathrm{Ag}^{+}$ions were the only cause of toxicity for Ag nanoparticles of this study was 


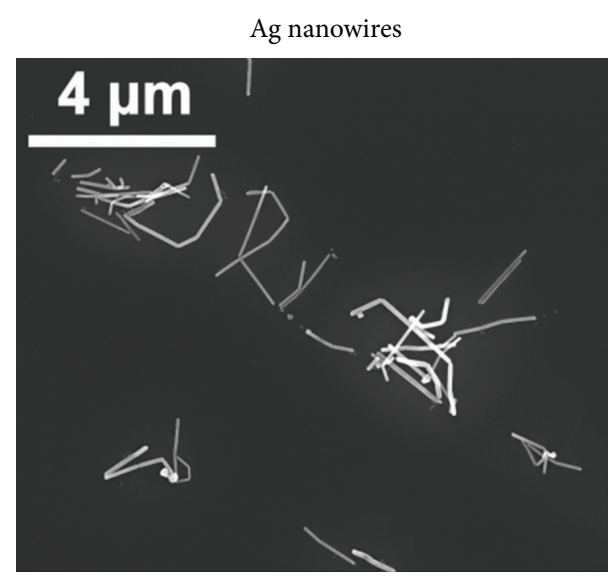

(a)

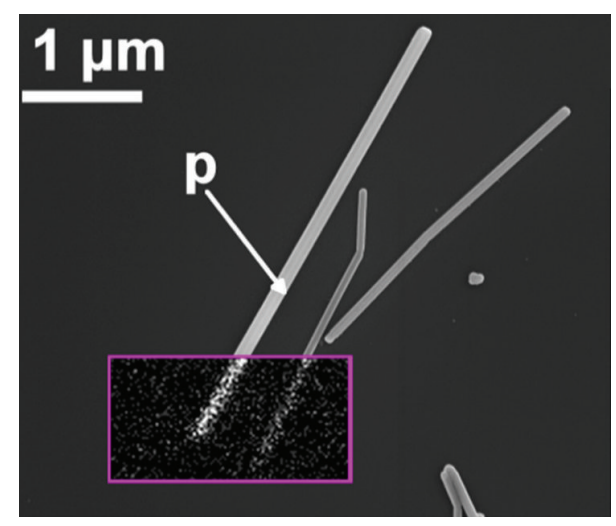

(c)

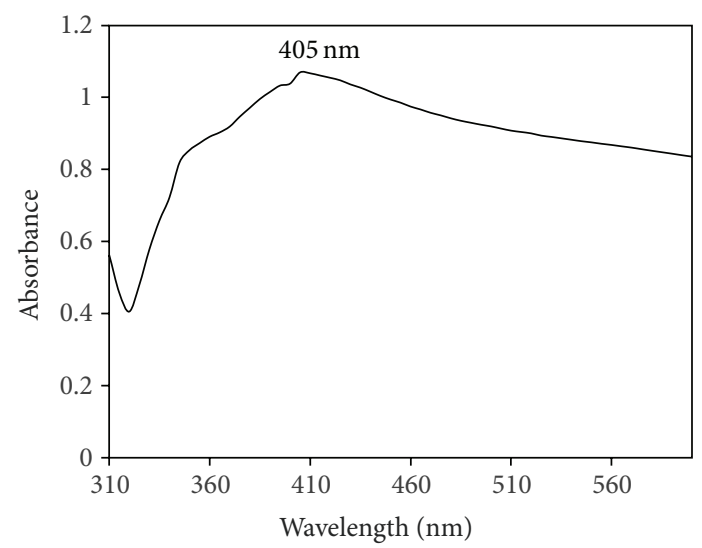

(e)

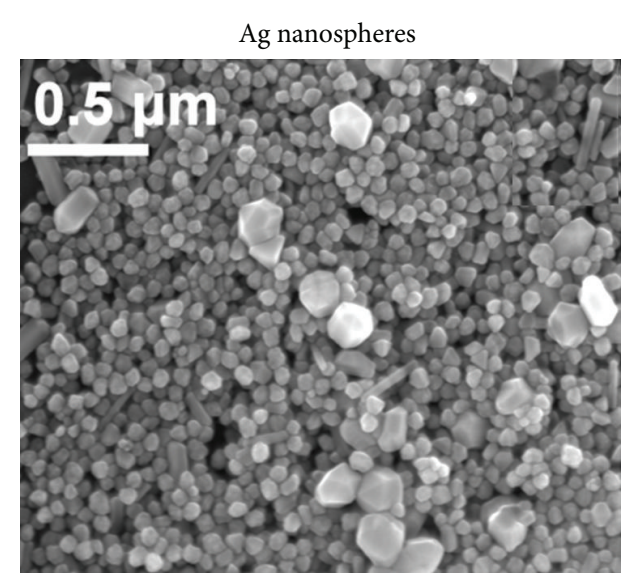

(b)

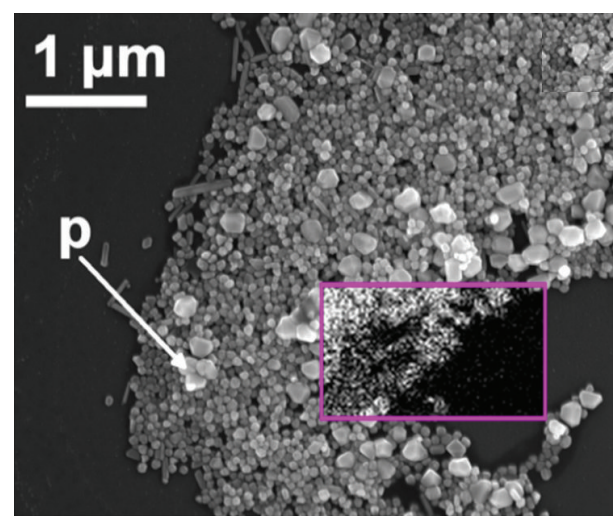

(d)

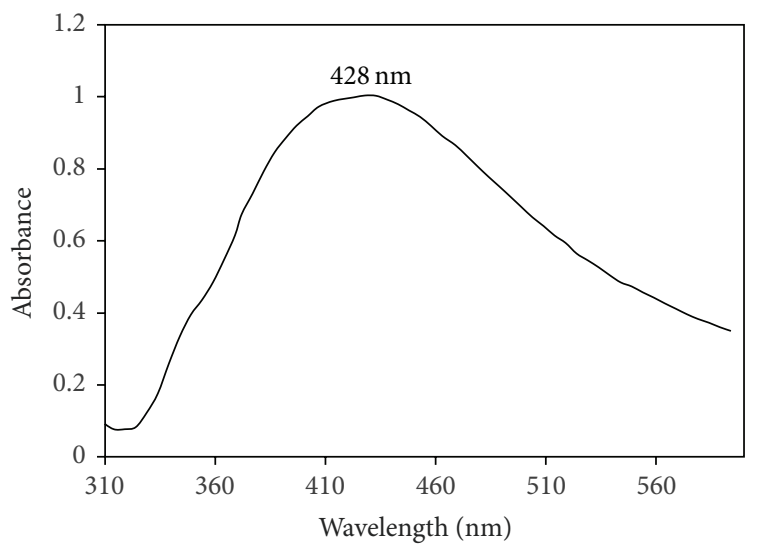

(f)

FIGURE 2: Scanning electron micrographs (SEM), EDX mapping, and UV-vis spectra of the Ag nanowires (NWs) and Ag nanospheres. (a) and (c) SEM micrographs of Ag NWs, (b) and (d) SEM micrographs of Ag nanospheres; note different magnifications; scale bars are indicated. Insets in (c) and (d) show EDX mapping. (e) UV-vis spectrum of Ag NWs and (f) UV-vis spectrum of Ag nanospheres. Maximum absorbance values are shown in (e) and (f).

obtained by comparing the toxicity of Ag NWs dispersion and its ultracentrifuged extract (Figure 4). According to bioluminescence inhibition assay, the EC50 values of noncentrifuged Ag NWs dispersion and its ultracentrifuged extract did not statistically significantly differ being $0.42 \pm 0.2$ and $0.75 \pm$ $0.25 \mathrm{mg} \mathrm{Ag/L}$, respectively. Similarly, no visually significant differences were observed when the decrease in bacterial cell count by Ag NWs suspension or ultracentrifuged extract was tested. In both cases remarkable decrease in bacterial cell count was observed at $3.8 \mathrm{mg} / \mathrm{L}$ (Figure 4(b)). Thus, both bioluminescence inhibition assay and viability assay indicated that the removal of Ag NWs from the test did not 
TABLE 2: Physicochemical characteristics of Ag nanospheres and nanowires (NWs).

\begin{tabular}{lcccc}
\hline & Primary size $^{1}, \mathrm{~nm}$ & $\zeta$-potential $^{2}, \mathrm{mV}(\mathrm{pH})$ & Hydrodynamic size $^{3}, \mathrm{~nm}^{(\mathrm{pdi})}$ & Dissolution $^{4}, \%$ \\
\hline Ag NWs & $100 \pm 40 \times 6100 \pm 2700$ & $-46(7.0)$ & Not relevant $^{5}$ & 2.4 \\
Ag spheres & $83 \pm 37$ & $-36(7.2)$ & $98 \pm 1.8(0.25)$ & 2.2 \\
\hline
\end{tabular}

${ }^{1}$ Determined from scanning electron micrographs; $n=20$.

${ }^{2}$ Analyzed using electrophoretic light scattering method. The data were analyzed using Smoluchowski approximation.

${ }^{3}$ Hydrodynamic size is based on dynamic light scattering (DLS) measurement. pdi: polydispersity index.

${ }^{4} \%$ nanomaterial dissolved was analyzed from ultracentrifuged extracts of $36 \mathrm{mg} / \mathrm{L}$ (Ag spheres) or $15 \mathrm{mg} / \mathrm{L}$ (Ag nanowires) dispersions by GF-AAS.

${ }^{5}$ Dynamic light scattering (DLS) measurement is not relevant for rod-shaped particles.

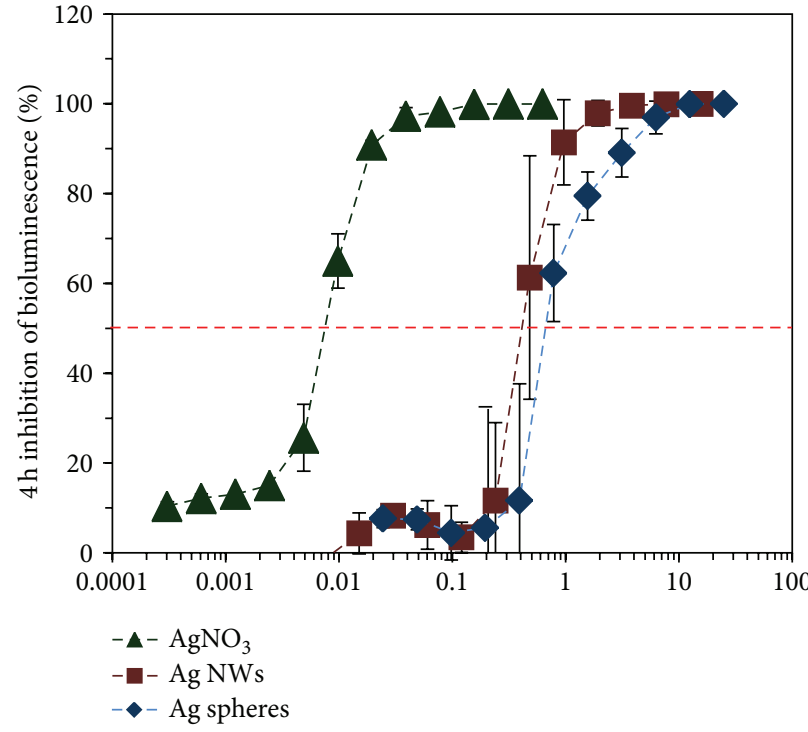

(a)

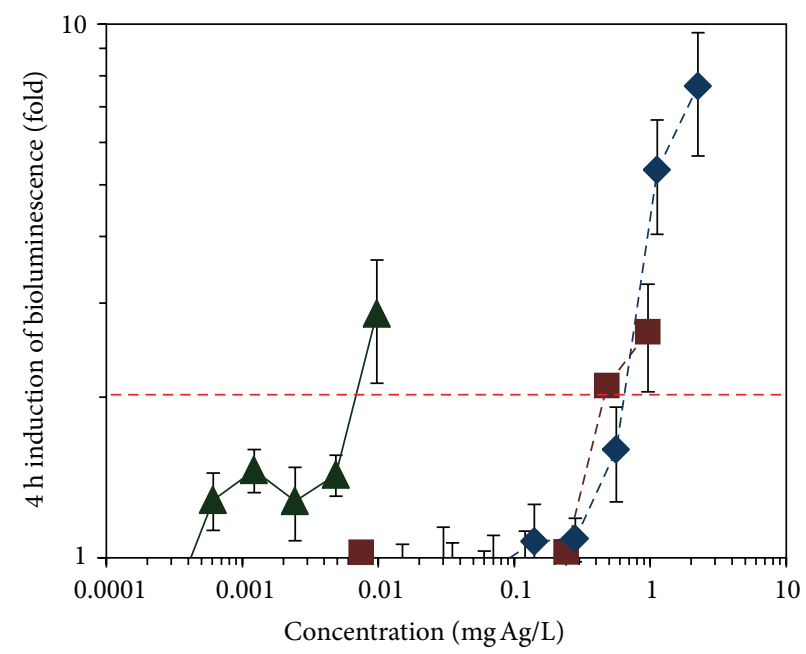

(c)

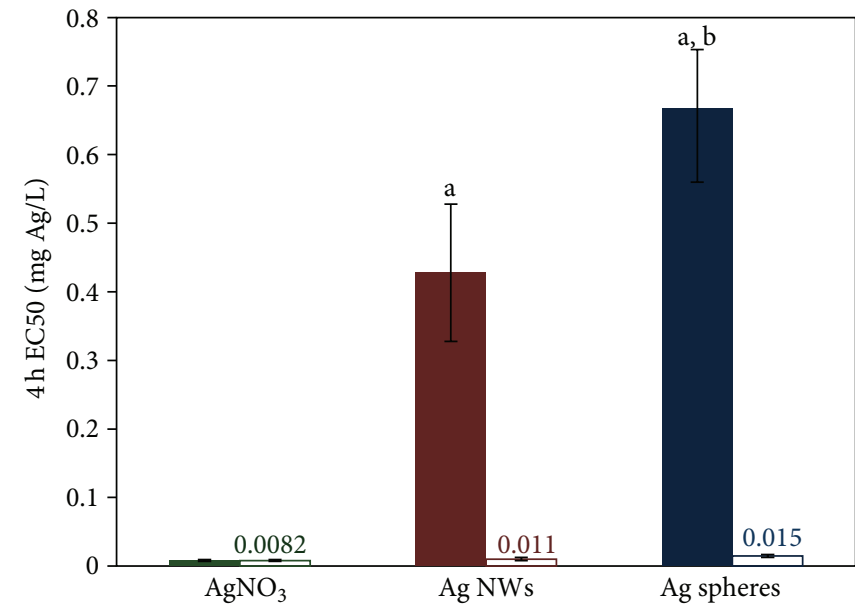

Nominal Ag

Dissolved Ag

(b)

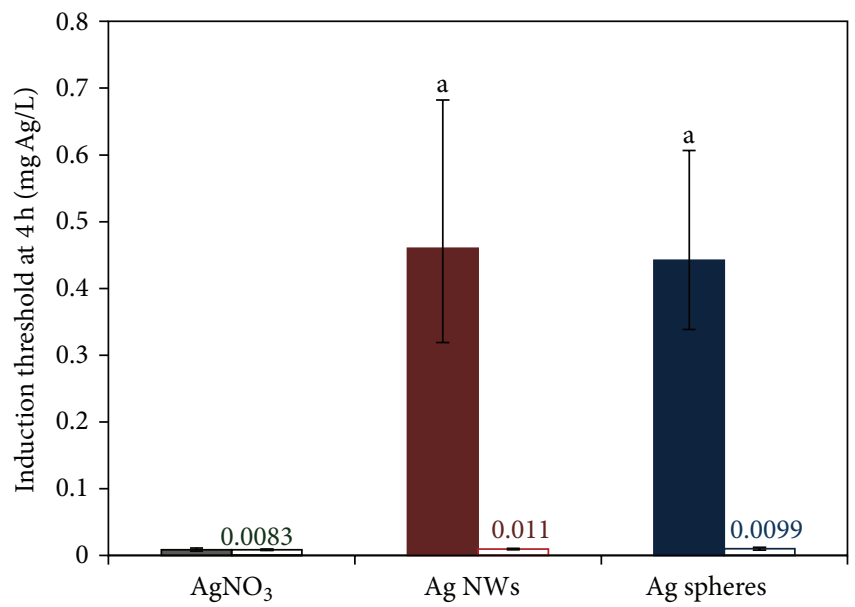

(d)

FIGURE 3: Dose-dependent effects of Ag nanowires (NWs), Ag nanospheres, and $\mathrm{AgNO}_{3}$ on two recombinant Escherichia coli strains after 4h exposure. (a) Inhibition of the light output in bioluminescent E. coli; (c) induction of the bioluminescence of the $\mathrm{Ag}^{+}$-induced E. coli. Filled columns in (b) indicate EC50 values calculated from panel (a) (see horizontal dotted line); filled columns in (d) indicate induction threshold values calculated from panel (c) (see horizontal dotted line). Open columns in (b) and (d) with numerical values show mg of dissolved Ag/L at EC50 or induction threshold, respectively. Mean \pm standard deviation of two independent experiments is shown: a-significant $(P<0.05)$ difference from $\mathrm{AgNO}_{3}$, b-significant $(P<0.05)$ difference from $\mathrm{AgNO}_{3}$ and $\mathrm{Ag} \mathrm{NW}$. 


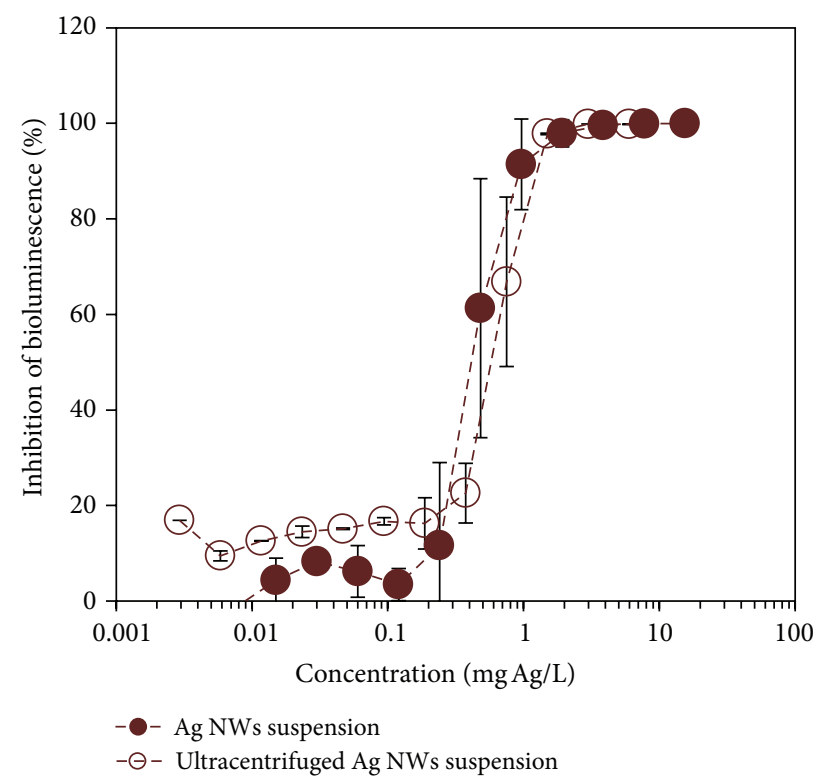

(a)

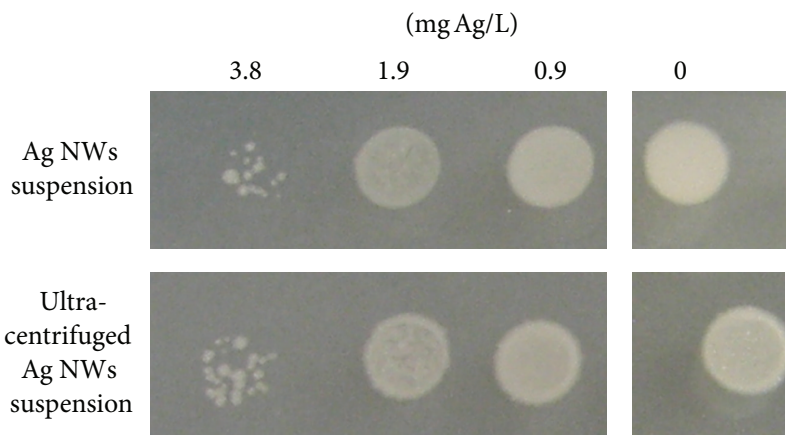

(b)

Figure 4: Antibacterial activity of Ag NWs and their particle-free supernatant to recombinant Escherichia coli. (a) Inhibition of bacterial bioluminescence by Ag NWs suspension (see also Figure 3(a)) and its supernatant (see Section 2). Mean \pm standard deviation of three independent experiments is shown; (b) potential of Escherichia coli to yield colonies on agarized LB growth medium after 4-hour incubation with different nominal concentrations of Ag NWs suspension and the respective particle-free supernatant. Photo is taken after $24 \mathrm{~h}$ incubation of the inoculated test plates at $30^{\circ} \mathrm{C}$. Opaque "spot" in (b) indicates high number of bacteria; appearance of individual colonies within the "spot" indicates decreased number of viable cells. All the Ag concentrations are nominal concentrations in Ag NWs suspension.

decrease the NWs toxicity and hence suggested that toxicity of $\mathrm{Ag} \mathrm{NWs}$ was indeed resulting from dissolved $\mathrm{Ag}^{+}$ions.

Comparison of EC50 values from bioluminescence inhibition assay and concentrations of Ag NWs that decreased the bacterial viability showed that the inhibition of bacterial bioluminescence occurred at 5-9-fold lower concentrations than were the concentrations that affected cellular viability. That is an additional proof that the inhibition of bacterial bioluminescence, either natural luminescent bacteria Vibrio fischeri or recombinant luminescent Escherichia coli, is an early indicator for toxicity that reflects the changes in bacterial energy metabolism [31] at a stage where viability is not yet affected. Indeed, bacterial bioluminescence has been shown to very significantly correlate with cellular viability [32], justifying its application in toxicity testing.

In summary, the results from this study indicate that the toxicity of $100 \times 6100 \mathrm{~nm} \mathrm{Ag}$ nanowires and $\mathrm{Ag}$ nanospheres with particle diameter of $83 \mathrm{~nm}$ occurred in very similar concentration range and was dictated solely by dissolved $\mathrm{Ag}^{+}$ ions. This finding is in agreement with the recent papers by Xiu et al. [14] and Bondarenko et al. [15] who also suggested that dissolution is the key element determining the toxicity of Ag nanostructures. On the other hand, according to our initial hypothesis and earlier papers by Pal et al. [16], Sadeghi et al. [17], and Ashkarran et al. [18], the shape of $\mathrm{Ag}$ nanostructures was also expected to play a role in Ag NPs antibacterial effects and toxicity. Thus, the fact that no shape-dependent toxicity was observed for nanowires and spheres was somewhat surprising. On the other hand, in previous studies reporting on shape-dependent toxicity of Ag nanoparticles comparisons were performed between particles that had relatively different particle size. For example, the diameter of $\mathrm{Ag}$ nanorods $(\sim 10 \mathrm{~nm})$ in the study by $\mathrm{Pal}$ et al. [16] was remarkably smaller than the size of $\mathrm{Ag}$ triangles $(\sim 40 \mathrm{~nm})$ that were studied. Also, the diameters of Ag spheres, triangles, wires, and cubes used by Ashkarran et al. [18] ranged from 17 to $92 \mathrm{~nm}$. Thus, in addition to specific effects of particle shape, the variable particle size may have also played a role in the differential toxicity of these particles. Furthermore, similarly to our study, no marked differences in antibacterial effects of Ag nanoparticles with slightly different shapes were reported by Sathishkumar et al. [33, 34]. Yet, it should be emphasized that the nanoparticles in our study were relatively large and our conclusion that antibacterial effects of Ag NPs do not depend on particles shape may not hold true in case of smaller nanoparticles. Clarification of this aspect requires further studies.

\section{Conclusions and Outlook}

In this study we showed that the toxicity of $100 \times 6100 \mathrm{~nm}$ (diameter $\times$ length, mean primary size) $\mathrm{Ag}$ nanowires and $83 \mathrm{~nm}$ nanospheres (mean primary size) to gram-negative bacterium E. coli-a representative of enteric pathogenswas driven by dissolved $\mathrm{Ag}^{+}$ions. Therefore, by regulating the solubilization of silver nanostructures, for example, by applying different coatings, it is possible to fine-tune the 
nanostructures towards higher toxicity, that is, to yield more efficient silver-based antibacterial agents, or, on the contrary, to render them less soluble if the main goal is other than antibacterial application (safe by design approach) [35].

Finally, although dissolution was shown to drive the toxicity of relatively large nanostructures as those studied in this paper, the possibility that the toxicity of smaller nanoparticles could also be driven by direct effects between nanoparticles and cells, that is, by "nano-specific" effects, cannot be excluded.

\section{Acknowledgments}

Financial support by the Estonian Research Council (Grants SF0690063s08, IUT2-25, ETF8561, and ETF8216), EU FP7 NanoValid Project (Contract 263147), Development Fund of University of Tartu, Estonian Nanotechnology Competence Center (EU29996), ERDF Projects ("IRGLASS" 3.2. 1101.12-0027, “TRIBOFILM” 3.2.1101.12-0028, "High-technology Materials for Sustainable Development” TK117, “Mesosystems-Theory and Applications" TK114), and Graduate School on Functional Materials and Technologies (ESF Project 1.2.0401.09-0079) is gratefully acknowledged. The authors are grateful to Mr. Rando Saar for help with FIBSEM-EDX setup and Dr. Margit Heinlaan for critically reviewing the paper.

\section{References}

[1] C. Marambio-Jones and E. M. V. Hoek, "A review of the antibacterial effects of silver nanomaterials and potential implications for human health and the environment," Journal of Nanoparticle Research, vol. 12, no. 5, pp. 1531-1551, 2010.

[2] M. C. Stensberg, Q. Wei, E. S. McLamore, D. M. Porterfield, A. Wei, and M. S. Sepúlveda, "Toxicological studies on silver nanoparticles: challenges and opportunities in assessment, monitoring and imaging," Nanomedicine, vol. 6, no. 5, pp. 879898, 2011.

[3] Y. Li, Y. Wu, and B. S. Ong, "Facile synthesis of silver nanoparticles useful for fabrication of high-conductivity elements for printed electronics," Journal of the American Chemical Society, vol. 127, no. 10, pp. 3266-3267, 2005.

[4] M. Law, L. E. Greene, J. C. Johnson, R. Saykally, and P. Yang, "Nanowire dye-sensitized solar cells," Nature Materials, vol. 4, no. 6, pp. 455-459, 2005.

[5] S. T. Dubas and V. Pimpan, "Humic acid assisted synthesis of silver nanoparticles and its application to herbicide detection," Materials Letters, vol. 62, no. 17-18, pp. 2661-2663, 2008.

[6] N. A. Melosh, A. Boukai, F. Diana et al., "Ultrahigh-density nanowire lattices and circuits," Science, vol. 300, no. 5616, pp. 112-115, 2003.

[7] A. Kahru and A. Ivask, "Mapping the dawn of nanoecotoxicological research," Accounts of Chemical Research, vol. 46, no. 3, pp. 823-833, 2012.

[8] I. Blinova, J. Niskanen, P. Kajankari et al., "Toxicity of two types of silver nanoparticles to aquatic crustaceans Daphnia magna and Thamnocephalus platyurus," Environmentai Science and Pollution Research, vol. 20, no. 5, pp. 3456-3463, 2013.

[9] J. Fabrega, S. N. Luoma, C. R. Tyler, T. S. Galloway, and J. R. Lead, "Silver nanoparticles: behaviour and effects in the aquatic environment," Environment International, vol. 37, no. 2, pp. 517531, 2011.

[10] C. M. Zhao and W. X. Wang, "Importance of surface coatings and soluble silver in silver nanoparticles toxicity to Daphnia magna," Nanotoxicology, vol. 6, no. 4, pp. 361-370, 2012.

[11] W.-R. Li, X.-B. Xie, Q.-S. Shi, H.-Y. Zeng, Y.-S. Ou-Yang, and Y.-B. Chen, "Antibacterial activity and mechanism of silver nanoparticles on Escherichia coli," Applied Microbiology and Biotechnology, vol. 85, no. 4, pp. 1115-1122, 2010.

[12] I. Sondi and B. Salopek-Sondi, "Silver nanoparticles as antimicrobial agent: a case study on E. coli as a model for Gramnegative bacteria," Journal of Colloid and Interface Science, vol. 275, no. 1, pp. 177-182, 2004.

[13] J. R. Morones, J. L. Elechiguerra, A. Camacho et al., "The bactericidal effect of silver nanoparticles," Nanotechnology, vol. 16, no. 10, pp. 2346-2353, 2005.

[14] Z. M. Xiu, Q. B. Zhang, H. L. Puppala et al., "Negligible particle-specific antibacterial activity of silver nanoparticles," Nano Letters, vol. 12, no. 8, pp. 4271-4275, 2012.

[15] O. Bondarenko, A. Ivask, A. Käkinen et al., "Particle-cell contact enhances antibacterial activity of silver nanoparticles," PLoS ONE, vol. 8, no. 5, Article ID e64060, 2013.

[16] S. Pal, Y. K. Tak, and J. M. Song, "Does the antibacterial activity of silver nanoparticles depend on the shape of the nanoparticle? A study of the gram-negative bacterium Escherichia coli," Applied and Environmental Microbiology, vol. 73, no. 6, pp. 17121720, 2007.

[17] B. Sadeghi, F. S. Garmaroudi, M. Hashemi et al., "Comparison of the anti-bacterial activity on the nanosilver shapes: nanoparticles, nanorods and nanoplates," Advanced Powder Technology, vol. 23, no. 1, pp. 22-26, 2012.

[18] A. A. Ashkarran, S. Estakhri, M. R. H. Nezhad et al., "Controlling the geometry of silver nanostructures for biological applications," Physics Procedia, vol. 40, pp. 76-83, 2013.

[19] S. Liu, L. Wei, L. Hao et al., "Sharper and faster "Nano darts" kill more bacteria: a study of antibacterial activity of individually dispersed pristine single-walled carbon nanotube," ACS Nano, vol. 3, no. 12, pp. 3891-3902, 2009.

[20] S. George, S. Lin, Z. Ji et al., "Surface defects on plate-shaped silver nanoparticles contribute to its hazard potential in a fish gill cell line and zebrafish embryos," ACS Nano, vol. 6, no. 5, pp. 3745-3759, 2012.

[21] L. C. Stoehr, E. Gonzalez, A. Stampfl et al., "Shape matters: effects of silver nanospheres and wires on human alveolar epithelial cells," Particle and Fibre Toxicology, vol. 8, article 36, 2011.

[22] J. A. Bearden, "X-ray wavelengths," Reviews of Modern Physics, vol. 39, no. 1, pp. 78-124, 1967.

[23] D. Chen, X. Qiao, X. Qiu, J. Chen, and R. Jiang, "Largescale synthesis of silver nanowires via a solvothermal method," Journal of Materials Science, vol. 22, no. 1, pp. 6-13, 2011.

[24] D. D. Evanoff Jr. and G. Chumanov, "Synthesis and optical properties of silver nanoparticles and arrays," ChemPhysChem, vol. 6, no. 7, pp. 1221-1231, 2005.

[25] X. Yang, J. Bai, Y. Wang et al., "Hydrogen peroxide and glucose biosensor based on silver nanowires synthesized by polyol process," Analyst, vol. 137, pp. 4362-4367, 2012.

[26] R. Ma, C. Levard, S. M. Marinakos et al., "Size-controlled dissolution of organic-coated silver nanoparticles," Environmental Science and Technology, vol. 46, no. 2, pp. 752-759, 2012. 
[27] A. Ivask, T. Rõlova, and A. Kahru, "A suite of recombinant luminescent bacterial strains for the quantification of bioavailable heavy metals and toxicity testing," BMC biotechnology, vol. 9, no. 1, p. 41, 2009.

[28] E. J. Calabrese, "Hormesis: a revolution in toxicology, risk assessment and medicine," EMBO Reports, vol. 5, supplement 1, pp. S37-S40, 2004.

[29] K. Hakkila, T. Green, P. Leskinen, A. Ivask, R. Marks, and M. Virta, "Detection of bioavailable heavy metals in EILAToxOregon samples using whole-cell luminescent bacterial sensors in suspension or immobilized onto fibre-optic tips," Journal of Applied Toxicology, vol. 24, no. 5, pp. 333-342, 2004.

[30] Y. Sun, Y. Yin, B. T. Mayers, T. Herricks, and Y. Xia, "Uniform silver nanowires synthesis by reducing $\mathrm{AgNO}_{3}$ with ethylene glycol in the presence of seeds and poly(vinyl pyrrolidone)," Chemistry of Materials, vol. 14, no. 11, pp. 4736-4745, 2002.

[31] I. Kurvet, A. Ivask, O. Bondarenko, M. Sihtmäe, and A. Kahru, "LuxCDABE-transformed constitutively bioluminescent Escherichia coli for toxicity screening: comparison with naturally luminous Vibrio fischeri," Sensors, vol. 11, no. 8, pp. 7865-7878, 2011.

[32] R. M. S. Thorn, S. M. Nelson, and J. Greenman, "Use of a bioluminescent Pseudomonas aeruginosa strain within an in vitro microbiological system, as a model of wound infection, to assess the antimicrobial efficacy of wound dressings by monitoring light production," Antimicrobial Agents and Chemotherapy, vol. 51, no. 9, pp. 3217-3224, 2007.

[33] M. Sathishkumar, K. Sneha, S. W. Won, C.-W. Cho, S. Kim, and Y.-S. Yun, "Cinnamon zeylanicum bark extract and powder mediated green synthesis of nano-crystalline silver particles and its bactericidal activity," Colloids and Surfaces B, vol. 73, no. 2, pp. 332-338, 2009.

[34] M. Sathishkumar, K. Sneha, and Y.-S. Yun, "Immobilization of silver nanoparticles synthesized using Curcuma longa tuber powder and extract on cotton cloth for bactericidal activity," Bioresource Technology, vol. 101, no. 20, pp. 7958-7965, 2010.

[35] O. Bondarenko, K. Juganson, A. Ivask et al., "Toxicity of $\mathrm{Ag}, \mathrm{CuO}$ and $\mathrm{ZnO}$ nanoparticles to selected environmentally relevant test organisms and mammalian cells in vitro: a critical review," Archives of Toxicology, vol. 87, no. 7, pp. 1181-1200, 2013. 

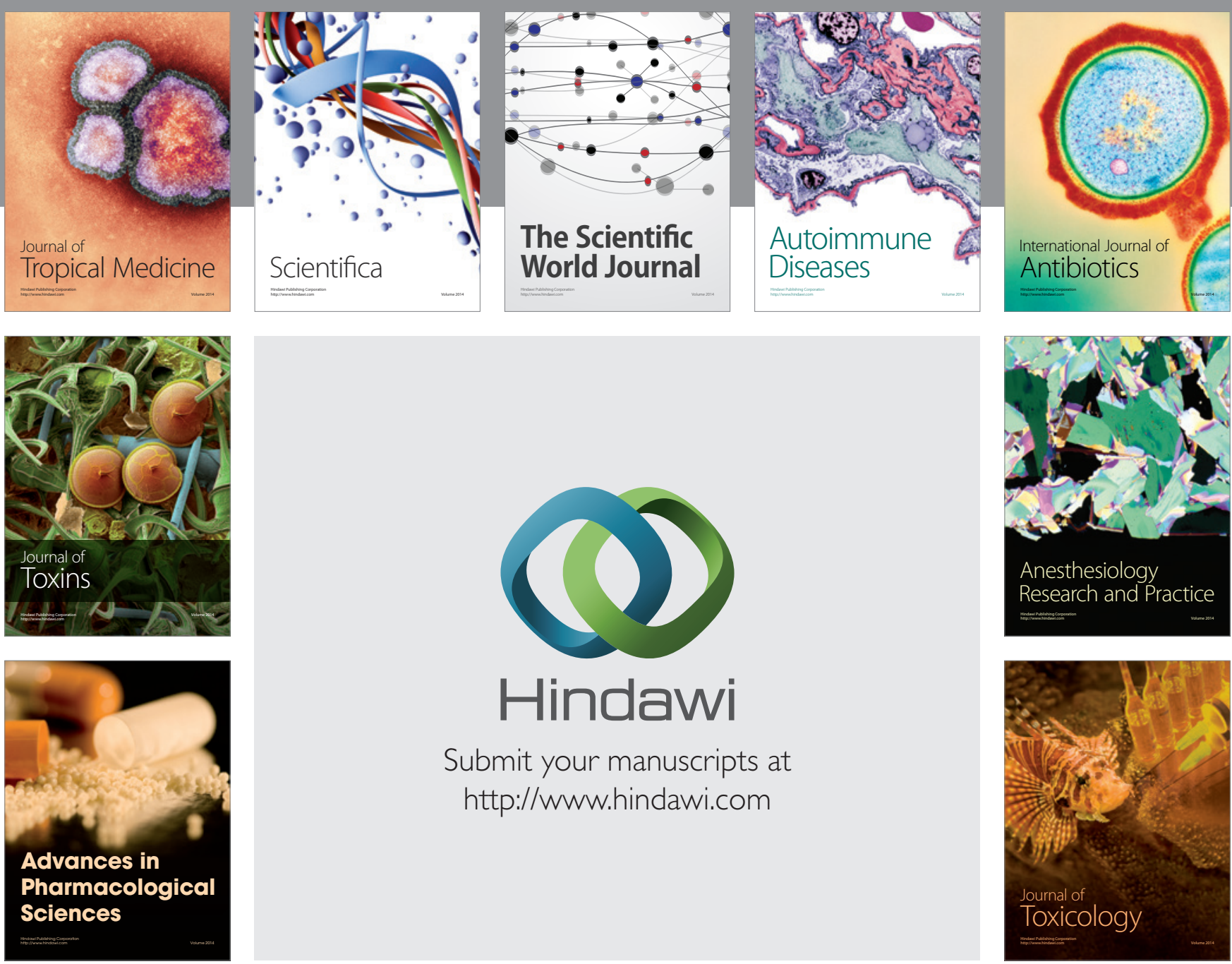

\section{Hindawi}

Submit your manuscripts at

http://www.hindawi.com
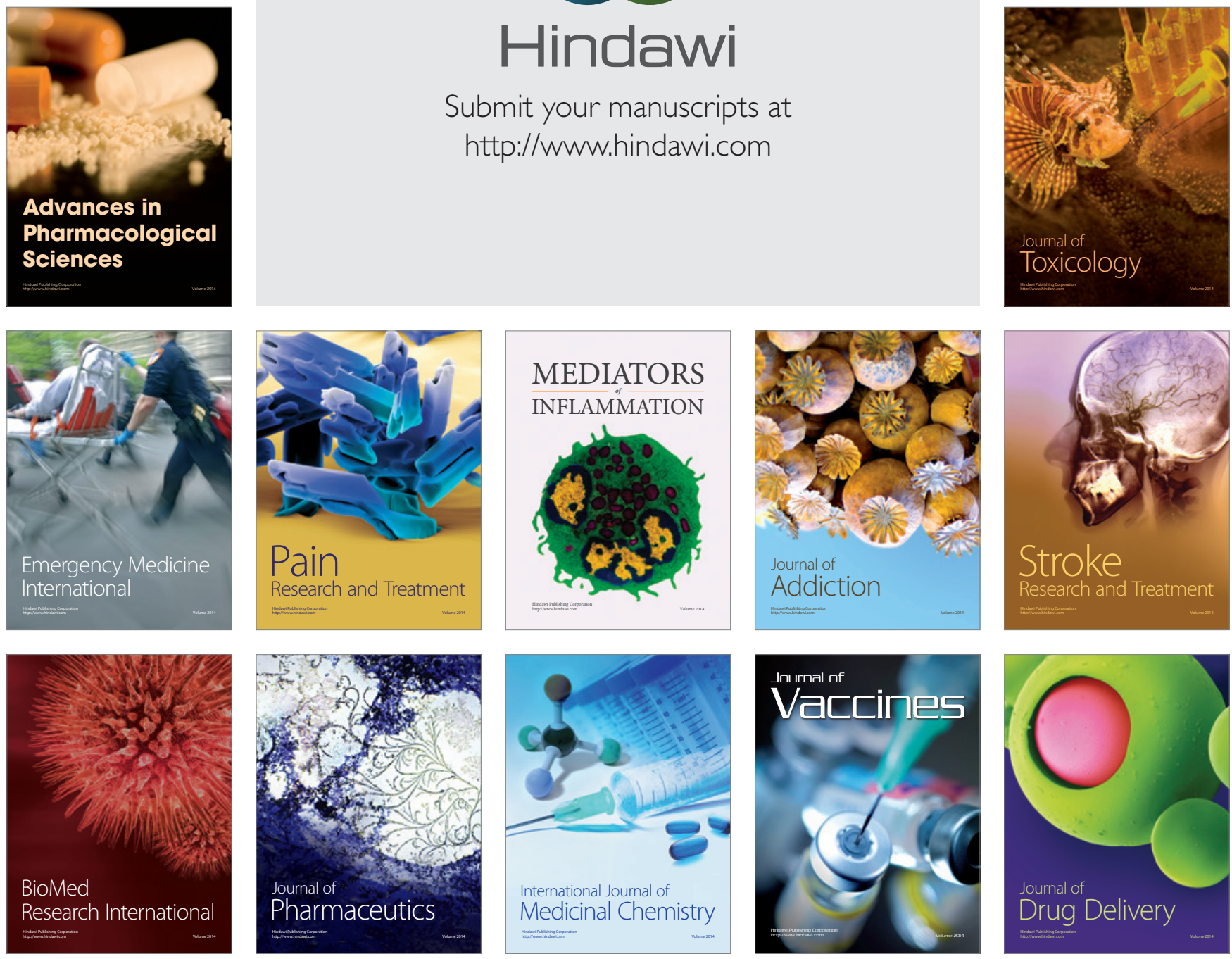